\title{
HYDRAULIC ANALYSIS OF LEAKAGE FROM SUBMARINE PIPE NETWORK
}

\author{
JunHua Li \\ YanTing Fan \\ School of Ocean Science and Engineering, Shanghai Maritime University \\ Shanghai, China
}

\begin{abstract}
The leakage from submarine pipe network is investigated by a numerical model in this paper. A whole event including of the steady flow before the leakage, the transient flow during the leakage and the steady flow after the leakage is considered in the model. The numerical results of the discharge and the head compare with the theoretical solution under the condition of steady flow. Good agreement is obtained. The numerical results of the discharge and the head show that the discharge and the head in each pipe changes suddenly after the leakage occurring. The abrupt trend of the discharge in each pipe is not same completely. Some change upwards, while others downwards. However, the abrupt trend of the head is always downward. The effects of leakage discharge on the transient flow are also investigated. It is founded that the larger the leakage discharge, the more obvious the abrupt trend of the discharge and the head before or after the leakage is.
\end{abstract}

Keywords: submarine pipe network, leakage, transient flow

\section{INTRODUCTION}

Submarine pipe networks are used as the important conveyance system all around the world. Unfortunately, the corrosion of the pipe, the interaction between wave current and networks and the mismanagement have led to leaking accidents in the submarine pipe networks. The leakage from submarine pipe networks will not only cause immense waste of resources and serious economic losses, but also severe pollution to the marine environment. Therefore, it is necessary to further the study of the leakage from submarine pipeline networks.

The submarine pipe network goes through from the initial state of no leaking to the leaking state and to the stable state after the leaking at last. Meanwhile, current in the pipes goes through from the initial steady state to the transient flow state and to the stable transient state at last. Therefore, these three states are not only mutual independent changing process but also mutual successive. In consequence, the flow process before and after the leakage should be studied as a successive changing process.

Previous researchers adopted steady flow model [6] and unsteady flow model $[5,7]$ to carry on researches about the inverse problem of network leakage respectively. Until now, there are less study that takes the before-leak state, transient leak state and stable leak state as a whole successive process. The reason may be that the previous networks put much emphasis on the maximum pressure caused by transient flow which results from equipment operations (like the sudden stop of pump or the start/stop of valve). The simulation before, in and after the process of leakage is important to the design, maintenance, operation and leak detection. Therefore, the point of this study is taking the state before the leakage, during the leakage and the stable leak state as a whole successive changing process of flow for simulation. The content includes the simulation of the whole process of the steady flow, the transient flow and the stable transient flow, the leakage influence towards the pressure and the discharge as well as the leakage magnitude influence towards the transient flow during the leakage.

\section{THE TRANSIENT FLOW MODEL OF PIPE NETWORKS}

\section{THE GOVERNING EQUATION}

The hydraulic parameters of the flow in the pipe change with time when the leakage occurs, which called the hydraulic transient. The property of hydraulic transient in the networks can be simulated by using transient flow model.

Assumed that the network is under a regular state before the leakage and there is no operation of equipments. At the moment, the flow of the network is in the stable state. After the stabilization of the leakage, the flow will also be under the condition of steady flow. So the steady flow model is used to stimulate before and after the leakage. Betâmio and Koelle [2] suggested that transient flow model can also be used to stimulate the steady flow state of the network. Therefore, this paper uses the transient flow model to stimulate the hydraulic behaviour before or after the leakage or during the leakage occurs. 
Assumed that the full pipe flow is in the network, in which liquid column separation won't occur. The pipe wall is made of linear elastic material. The frictional loss under the unsteady state equals to the one in the steady state. With all conditions above, the transient flow equation of one-dimensional network [8] can be expressed as:

$$
\begin{gathered}
\frac{\partial H}{\partial t}+\frac{a^{2}}{g A} \frac{\partial Q}{\partial x}=0 \\
\frac{\partial H}{\partial x}+\frac{1}{g A} \frac{\partial Q}{\partial t}+\frac{f Q|Q|}{2 g D A^{2}}=0
\end{gathered}
$$

When the leakage occurs in the network, the outflow equation of the leaking orifice is written as:

$$
Q_{L}=C_{d} A_{L} \sqrt{2 g\left(H_{L}-z_{L}\right)}
$$

Where: $\mathrm{x}$ is the distance along with the pipe; $\mathrm{t}$ is the time; $\mathrm{Q}$ is the discharge; $\mathrm{H}$ is the piezometric head; $\mathrm{D}$ is the inner diameter of pipe; $A$ is the cross sectional area of pipe; $a$ is the water hammer wavespeed; $g$ is the acceleration of gravity; $f$ is the Darcy-Weisbach coefficient of friction resistance; QL is the discharge of leakage; $\mathrm{Cd}$ is the coefficient of leaking flux; $\mathrm{AL}$ is the area of leakage; HL is the piezometric head in the pipe where leakage occurs; $\mathrm{zL}$ is the potential head where the leakage occurs.

\section{SOLUTIONS}

The transient flow equations are a group of quasi-linear hyperbolic partial differential equations, which including the continuity equation and the momentum equation. It only can be solved by numerical method. At present, method of characteristics (MOC) is most common to solve the transient flow in the networks [8]. The equations are discretized by the method of characteristics. The specific process of discretization is seen in document 1 or 8 . The equations after discretization are written as:

$$
\begin{array}{ll}
\left(H_{P}-H_{A}\right)+\frac{a}{g A}\left(Q_{P}-Q_{A}\right)+\frac{f \Delta x Q_{A}^{2}}{2 g D A^{2}}=0 & \frac{d x}{d t}=C^{+} \\
\left(H_{P}-H_{B}\right)-\frac{a}{g A}\left(Q_{P}-Q_{B}\right)-\frac{f \Delta x Q_{B}^{2}}{2 g D A^{2}}=0 & \frac{d x}{d t}=C^{-}
\end{array}
$$

Where the subscript $\mathrm{P}$ is present point at present time step, the subscript $A$ is the previous point at previous time step; the subscript $\mathrm{B}$ is the latter point at the previous time step; $\Delta \mathrm{x}$ is space intervals, $\mathrm{C}+$ and $\mathrm{C}$ - is the characteristic line and the inverse characteristic line respectively.

There are four unknown for each section in the pipe networks: upstream flux, upstream head, downstream flux and downstream head. These four unknowns are not solved by those two equations mentioned above. It still needs two compatible equations, which include equations of the mass conservation and the equivalence of head at the nodes. These two compatible equations are not used on the boundary. As a result, the flux, the head or the relation between flux and head are given. The simultaneous equations (discrete equations, compatible equations and boundary conditions) are shown as the form of matrix:

$$
[M]\{v\}=\{R\}
$$

Where $[\mathrm{M}]$ is the coefficient matrix of unknown variables, $\{\mathrm{v}\}$ is an unknown vector of the discharges and the heads at present time step, $\{R\}$ is a vector independent on unknown variables.

\section{THE STEADY FLOW MODEL IN PIPE NETWORKS}

The initial hydraulic parameters (velocity and head) are given to solve the transient flow model. In some pipe systems, the maximal pressure under the condition of transient flow is quite sensitive to the velocity under the initial condition [4]. Therefore, it is significant to give reasonable flow velocity and pressure under the initial steady flow condition for the calculation of transient flow.

With regard to an operational pipe network, the flux in each pipe and the head in the node are obtained by the solution of the steady flow model in pipe networks according to the boundary conditions and topology structure of the network. The following steady flow model is used to solve the flux and the head in the initial condition.

\section{CONTINUITY EQUATION AT THE NODE}

$$
\sum_{\mathrm{n}=1}^{j} Q_{i n}+Q_{i q}=0 \quad i=1,2, \cdots, M
$$

Where $\mathrm{M}$ is the number of nodes, $\mathrm{i}$ is number of the pipe connected with the node $\mathrm{i}$; Qiq is the external flux at the node i. It sets that the flux into the node is positive and the flux out the node is negative.

\section{ENERGY EQUATION}

$$
\left|H_{i}^{k}-H_{j}^{k}\right|=\left|\bar{R} Q_{\mathrm{k}}^{2}\right| \quad k=1,2, \cdots, L
$$

Where $\mathrm{L}$ is the number of pipes, $\mathrm{k}$ is the number of pipe connecting between node $i$ and node $j$,

$$
\bar{R}=\frac{8 \lambda l_{k}}{\pi^{2} d_{k}^{5} g}
$$

Hardy-Cross Method and Newton-Raphson Iterative Method are used as the solutions to the equations mentioned above. Hardy-Cross Method is available for loop pipe network (if none-loop pipe network, forming loop network by virtual pipes). Newton-Raphson Iterative Method is used in this paper. 


\section{APPLICATION}

It is stimulated the process of leakage in the pipe network shown in Fig.1. This pipe network was introduced by Pudar and Liggett originally [6], and then by Ligget and Chen [5]. The lengths of the pipes in this pipe network are given in the figure. The pipe diameter is $254 \mathrm{~mm}$. The thickness of pipe is $1.6 \mathrm{~mm}$. Node 1 and node 4 are boundary nodes. Node 1 is connected with the tank, with $30 \mathrm{~m}$ head constantly. Node 4 is the outflow node, with $0.01 \mathrm{~m} 2 / \mathrm{s}$ quantity of flux

\section{THE CONDITION OF STEADY FLOW}

To verify the program, calculate the pipe network under the condition of steady flow firstly. Tab.1 shows the flux in each pipe. The results compare with the theory solutions by taking node 3 as an example. The pipes connecting with node 3 are pipe 2, pipe 3, pipe 10 and pipe 11 as shown Fig.1. Suppose that the discharge into node 3 is positive, and outflow is negative. Plus all the discharge at node 3 and get: . Similarly, the sum of node $2,4,5,6$ is $0.0,-0.001,0.0,0.0,0.0$ respectively. The results above are approximate to the theory solution of the accumulative sum of node flux being 0 in the condition of steady flow.

Tab.1. The velocity and discharge in each pipe under the condition of steady flow

\begin{tabular}{cccccc}
\hline Pipe No. & $\begin{array}{c}\text { Upstream } \\
\text { node }\end{array}$ & $\begin{array}{c}\text { Downstream } \\
\text { node }\end{array}$ & $\begin{array}{c}\text { Pipe length } \\
(\mathrm{m})\end{array}$ & $\begin{array}{c}\text { Velocity } \\
(\mathrm{m} / \mathrm{s})\end{array}$ & $\begin{array}{c}\text { Discharge } \\
\left(\mathrm{m}^{3} / \mathrm{s}\right)\end{array}$ \\
\hline 1 & 1 & 2 & 1066.8 & 0.92 & 0.047 \\
2 & 1 & 3 & 762 & 1.05 & 0.054 \\
3 & 3 & 2 & 762 & 0.30 & 0.015 \\
4 & 2 & 5 & 1066.8 & 0.21 & 0.011 \\
5 & 2 & 4 & 762 & 1.01 & 0.051 \\
6 & 5 & 4 & 762 & 0.96 & 0.048 \\
7 & 6 & 5 & 762 & 0.15 & 0.007 \\
8 & 7 & 6 & 762 & 0.15 & 0.007 \\
9 & 7 & 5 & 1066.8 & 0.18 & 0.009 \\
10 & 3 & 7 & 762 & 0.33 & 0.016 \\
11 & 3 & 5 & 762 & 0.41 & 0.021 \\
\hline
\end{tabular}

Tab. 2 shows the head at each node in pipe network under the condition of steady flow. The numerical results compare with the solutions of Darcy-Weisbach Equation. The coefficient of friction resistance is obtained by Swamee-Jain Equation. It is known from the table that the head differences of pipe between two nodes are basically agreement with the solution of Darcy-Weisbach Equation.

Tab.2. The head at the nodes

\begin{tabular}{ccccccc}
\hline Pipe No. & $\begin{array}{c}\text { Head at } \\
\text { usstream } \\
\text { node }\end{array}$ & $\begin{array}{c}\text { Head at } \\
\text { downstream } \\
\text { node }\end{array}$ & $\begin{array}{c}\text { Head } \\
\text { difference } \\
(\mathrm{m})\end{array}$ & $\begin{array}{c}\text { Velocity } \\
(\mathrm{m} / \mathrm{s})\end{array}$ & $\mathrm{f}$ & $h_{f}=f \frac{l}{d} \frac{V^{2}}{2 g}$ \\
\hline 1 & 30.00 & 27.17 & 2.83 & 0.92 & 0.0155 & 2.81 \\
2 & 30.00 & 27.44 & 2.56 & 1.05 & 0.0151 & 2.54 \\
3 & 27.44 & 27.17 & 0.27 & 0.30 & 0.0195 & 0.27 \\
4 & 27.17 & 26.96 & 0.21 & 0.21 & 0.0210 & 0.20 \\
5 & 27.17 & 24.78 & 2.39 & 1.01 & 0.0152 & 2.37 \\
6 & 26.96 & 24.78 & 2.18 & 0.96 & 0.0154 & 2.17 \\
7 & 27.04 & 26.96 & 0.08 & 0.15 & 0.0228 & 0.08 \\
8 & 27.12 & 27.04 & 0.08 & 0.15 & 0.0228 & 0.08 \\
9 & 27.12 & 26.96 & 0.16 & 0.18 & 0.0218 & 0.15 \\
10 & 27.44 & 27.12 & 0.32 & 0.33 & 0.0191 & 0.32 \\
11 & 27.44 & 26.96 & 0.48 & 0.41 & 0.0182 & 0.47 \\
\hline
\end{tabular}

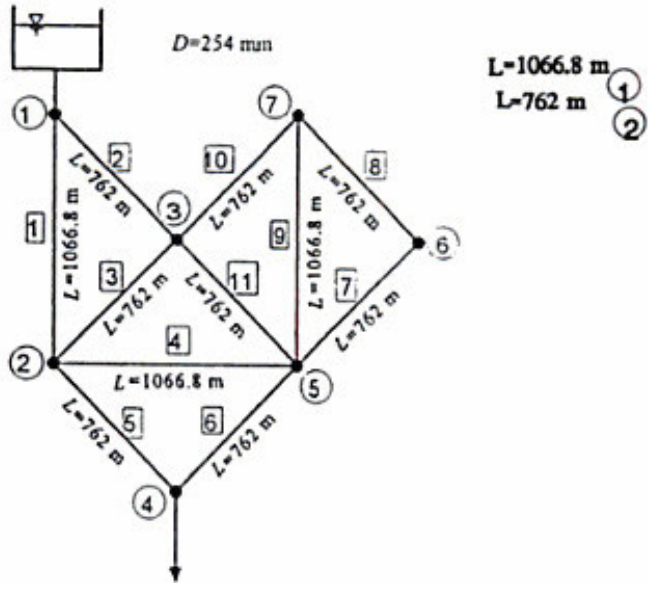

Fig. 1 The process of leakage in the pipe network

\section{THE CONDITION OF LEAKAGE}

\section{LEAKAGE AT NODE 5}

The number is maximum pipes at node 5 as shown in Fig.1. If leakage occurs at node 5 , the variation of the flux or head in all pipes is quite sensitive to the leakage from other nodes. In consideration of this aspect, the leaking at node 5 is considered firstly.

(1) The condition of a sudden leakage

Simulation of the transient flow resulted from the leakage at node 5 . The total length of time is 578 s. From 0 s- 110 s, there is no leaking in steady flow. At 111st s, the leakage occurred at node 5 , with $41-\mathrm{mm}$ diameter leaking orifice, 61 pipe sections, 57 nodes and 0.115 s time step.

Fig. 2 shows the downstream discharge variation with time in 11 pipes. From the figures, it is obtained that the downstream flux in pipe 6 (namely the flux of node 4 ) remain steady under the condition of steady flow and suddenly decrease after the leakage compared with the one in the steady state. After a period of time, the discharge became steady again. However, the abrupt trends of downstream discharges in other pipes are opposite to the one in pipe 6 after the leakage occurs.

Fig. 3 gives the node 2-7 head variation with the time respectively. As shown in the figures, the heads of all nodes are constant before the leakage occurred. When the leakage occurred, the head changed suddenly until steady. The head after the leakage is less than the one in initial steady flow.

With regard to the leakage sensitivity of flux and head, the figures show clearly that the flux in the pipes is more sensitive to the leakage than the head.

Fig.4 gives the partial enlarged drawing of the head variation with time at node 4 when the transient flow occurred. As shown in the figure, the head waved periodically in transient flow. The period and the peak amplitude of waves decrease gradually. The peak amplitude of head is 1.3 times of the one in the condition of steady flow. The minimum value of head is 1.5 times of the one under the condition of steady.

(2) The condition of a slow leakage

Besides the speed of leak slows down, other conditions remain the same. The leak occurs from the 111st s, with the 


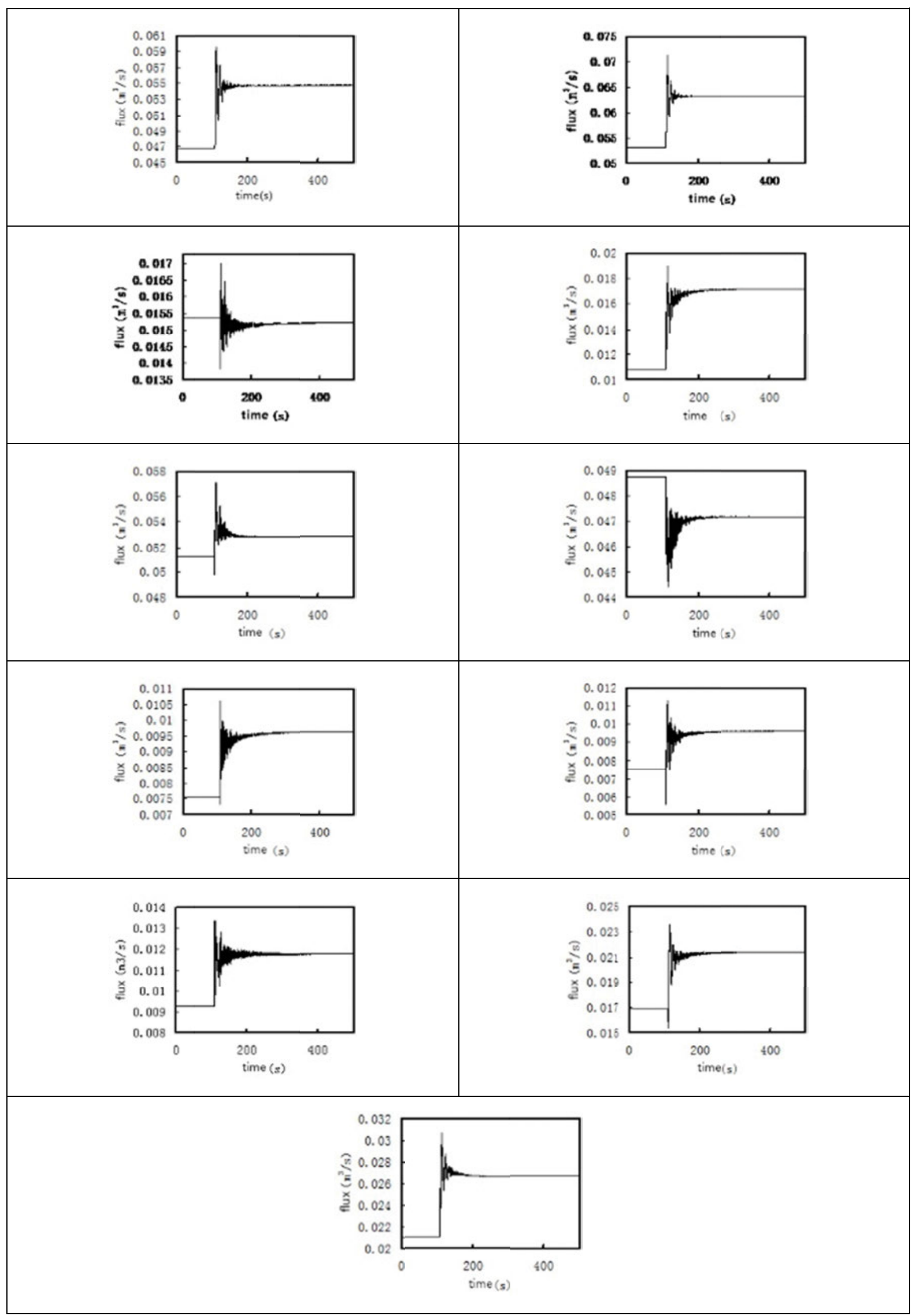

Fig. 2 The downstream discharge variation with time in 11 pipes 


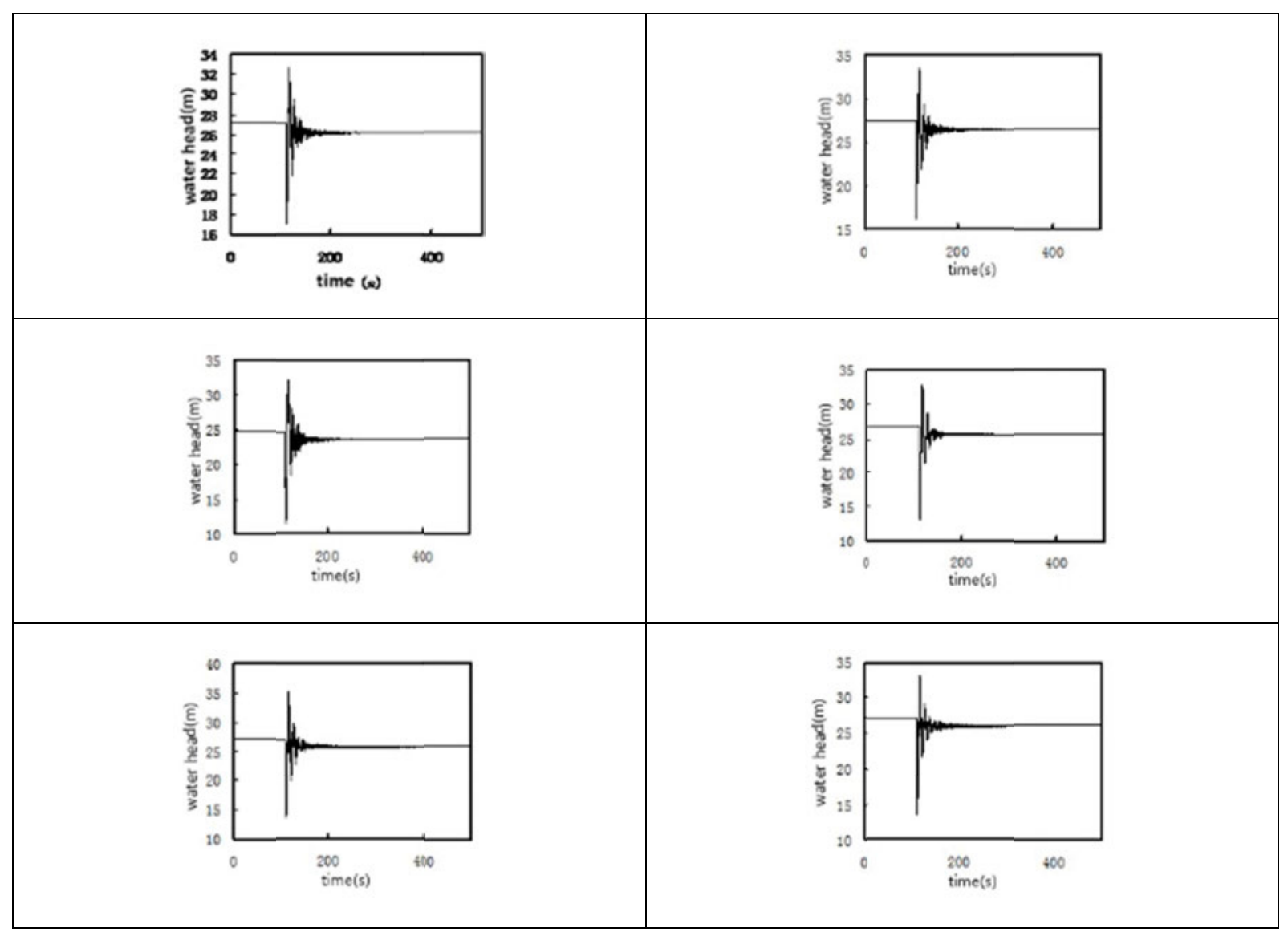

Fig. 3 The node 2-7 head variation with the time respectively

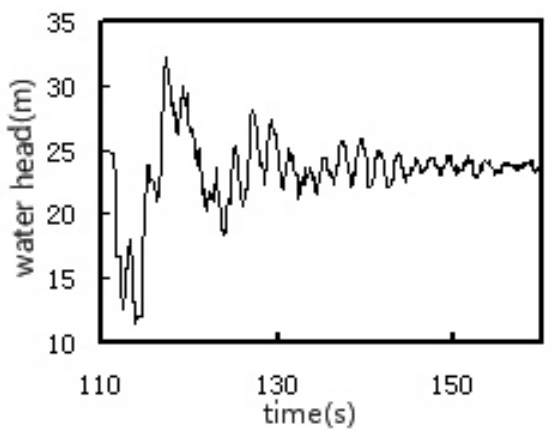

Fig. 4 The partial enlarged drawing of the head variation with time at node 4

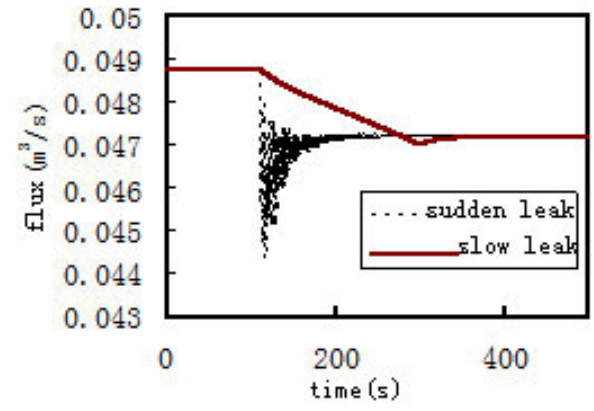

Fig. 6 The downstream discharge variation with time in the pipe 6

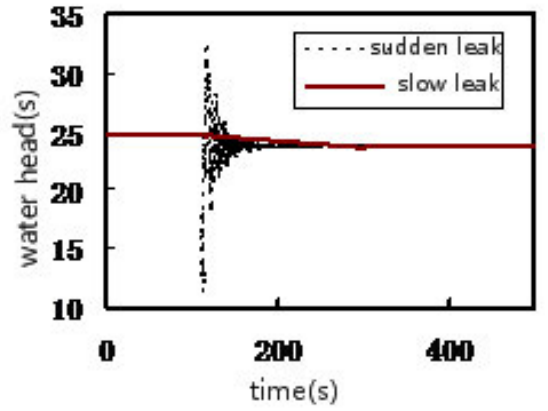

Fig. 5 The head variation with the time at the node 4

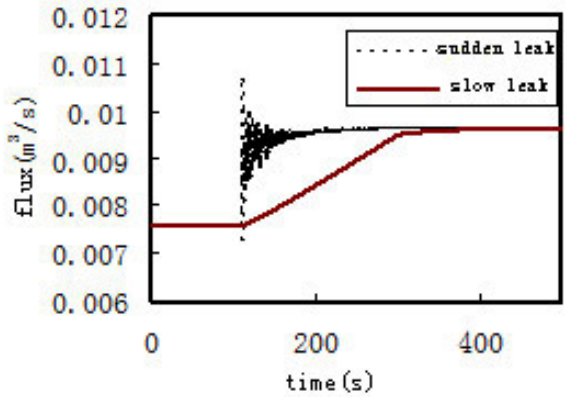

Fig. 7 The downstream discharge variation with time in the pipe 7 


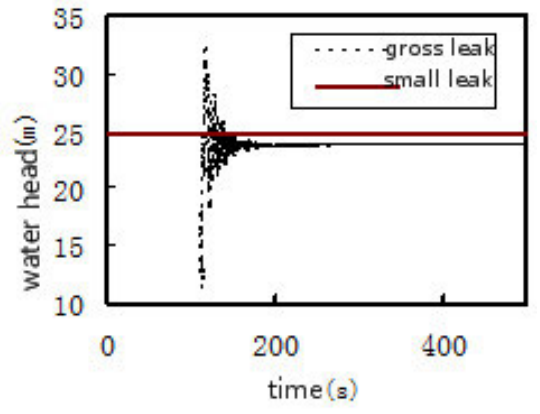

Fig. 8 The head variation with time at node 6 with the diameter $41 \mathrm{~mm}$ and $15 \mathrm{~mm}$ respectively
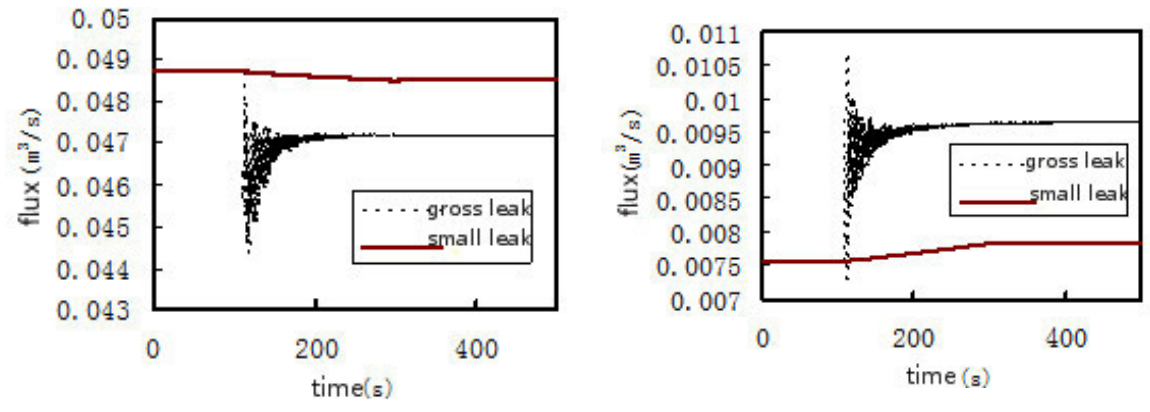

Fig. 9 The flux variation with time under the condition of the leakage diameter $41 \mathrm{~mm}$ and $15 \mathrm{~mm}$ respectively

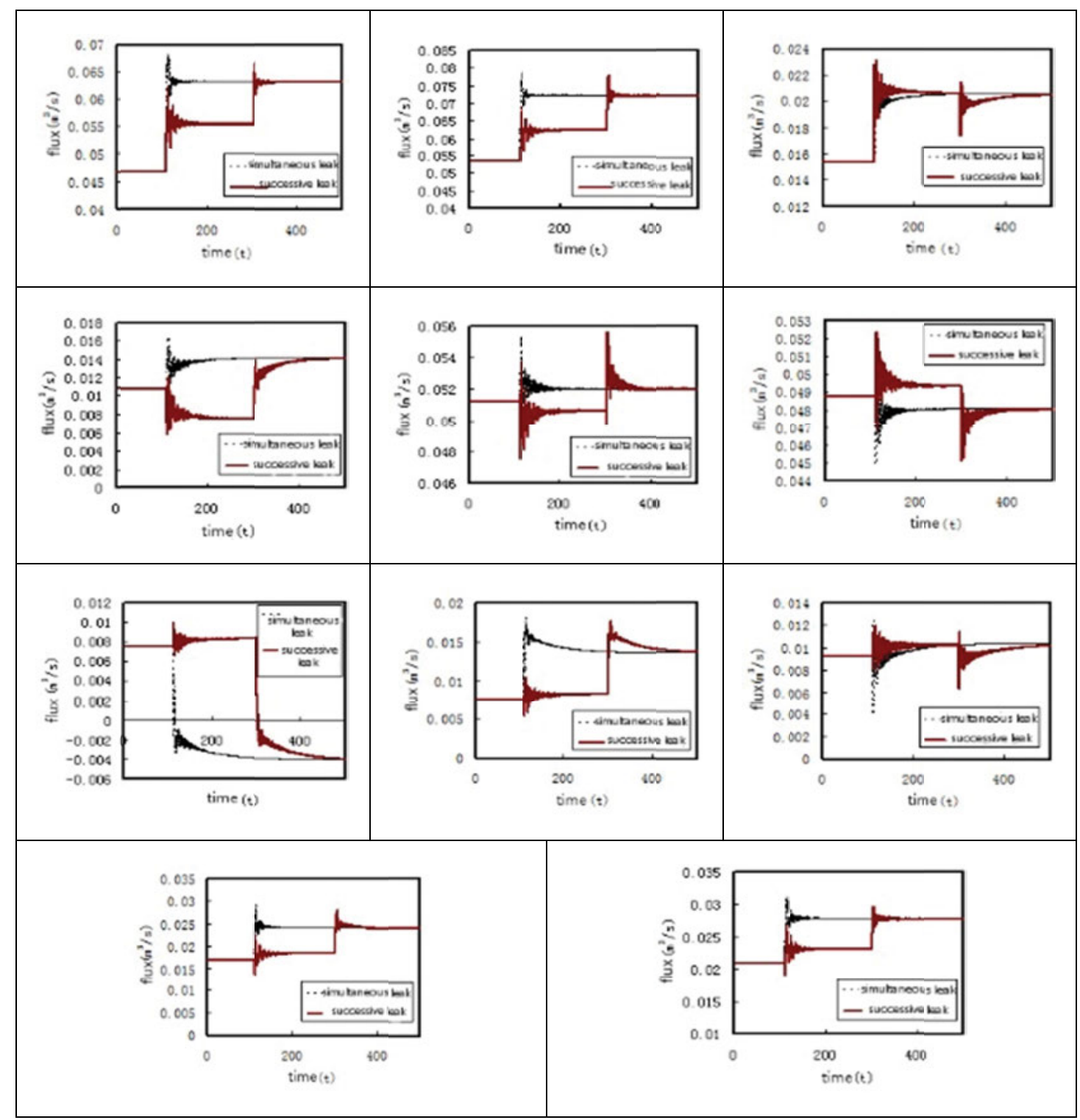

Fig. 10 The variation of downstream flux in the pipes and head at nodes with time under the condition of simultaneous and successive leakage respectively 
diameter of leaking orifice increased from 0 gradually to $41 \mathrm{~mm}$ at the 300th s.

The result also shows that the change law of flux and head in each pipe under the condition of the slow leakage is same as the one of a sudden leakage. However, the fluctuation range in transient flow is various tremendously.

Fig. 5 gives the head variation with the time at the node 4 under the condition of sudden and slow leakage respectively. As shown in the figure, the head is slightly fluctuated during the slow leakage. There is no obvious peak and trough. So the slow leakage does not cause tremendous hammer pressure.

Fig. 6 and Fig. 7 gives the downstream discharge variation with time in the pipe 6 and 7 respectively. It is also shown in the figure that the changing regulation is similar to the one of the head. Namely the flux fluctuation is not obvious when a slow leakage occurs.

(3) Different diameters of leakage

Suppose that the diameter of leakage is $15 \mathrm{~mm}$. The other condition is same as the condition of sudden leakage. Fig. 8 shows the head variation with time at node 6 with the diameter $41 \mathrm{~mm}$ and $15 \mathrm{~mm}$ respectively. Fig.9 gives the flux variation with time under the condition of the leakage diameter $41 \mathrm{~mm}$ and $15 \mathrm{~mm}$ respectively. These figures show clearly that the head, the flux and the intensity of fluctuation under the condition of the small leak are all less than the ones under the condition of the large leak.

\section{LEAKAGE AT OTHER NODES}

All the examples above analyze the flux and the head

Tab 3. The abrupt trend of downstream flux when leakage occurs at different nodes

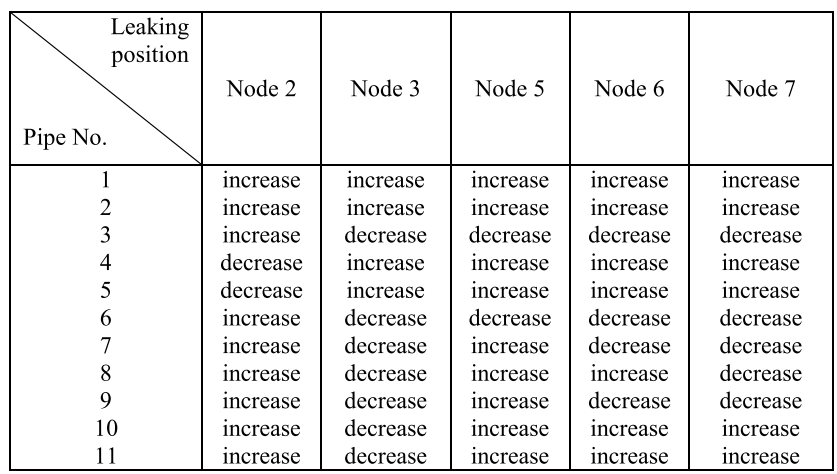

Tab.4. The abrupt trend of the head when leakage occurs at different nodes

\begin{tabular}{|l|l|l|l|l|l|}
\hline $\begin{array}{l}\text { Leaking } \\
\text { position }\end{array}$ & Node 2 & Node 3 & Node 5 & Node 6 & Node 7 \\
\hline 2 & & & & \\
\hline 3 & decrease & decrease & decrease & decrease & decrease \\
Pipe No. & decrease & decrease & decrease & decrease & decrease \\
& decrease & decrease & decrease & decrease & decrease \\
decrease \\
5
\end{tabular}

variation when a leakage occurs at node 5 . How change the flux and the head when the leakage occurs at other nodes? Is it the same as the one when a leakage occurred at node 5 ? In consideration of this aspect, it is investigated the condition that the leakage occurs at other node except node 5 . The time length and the leakage condition are same as those of the sudden leakage at node 5 .

Tab. 3 and Tab. 4 show the changing trend of the downstream flux in all pipes and the head at all nodes before and after the leakage occurs at other nodes. Table 3 points out that the combined changing laws of downstream flux in all pipes under the condition of the leakage at different node are different. Meanwhile, there is no repeatability in the combined changing forms. From Table 4, it is pointed out that no matter which nodes there is leakage, the node head is less than the one in steady flow without leakage.

\section{MULTI-NODES LEAKAGE}

The leakage in the pipe network may be occurred at multiple nodes. Multi-node leakages may occur simultaneously, or occur successively. Thus, it is investigated the condition that the leakage occurs at two nodes simultaneously and occurs successively. The leaked positions are chosen at node 2 and node 6 . The leakage condition is same as that of a sudden leakage at node 5 . The leakage began at node 2 firstly and then at node 6 after leaking for 190s under the condition of the successive leakage. The total time of leakage still is $578 \mathrm{~s}$.

Fig.10 show the variation of downstream flux in the pipes and head at nodes with time under the condition of simultaneous and successive leakage respectively. From the view, the flux goes up and down compared with the steady flow without leakage, while the head declines. In comparison with simultaneous leakages, the changing trend of the head and flux before and after the leakage is the same. Moreover, the flux and head exists the periodical abrupt changes in the process of leakage.

\section{CONCLUSIONS}

The transient flow model is employed for simulating the leakage in pipe network. The whole event including of the steady flow before the leakage, the transient flow during the leakage and the steady flow after the leakage is considered in the model. The numerical results are validated against the theoretical solution under the condition of steady flow. The following conclusions are obtained:

(1) When the leakage occurs in the pipes, the flux and the head change suddenly and fluctuate, in comparison with those before the leakage. The abrupt trend of the flux in each pipe is not same completely. Some is upwards, while others downwards. However, the abrupt trend of the head is always downwards.

(2) The fluctuation strength of the flux and the head under the condition of the leakage is related with the magnitude and speed of the leakage. As it leaks more and faster, the fluctuating 
range of the head and the flux is bigger.

(3) When the leakages occur in different position, the combination law of abrupt trend of the flux is different. The abrupt trend of the head is downward no matter where the position of the leakage.

(4) When the leakages occur successively, the abrupt change of the flux and the water head are all stepped.

\section{ACKNOWLEDGMENTS}

This paper was supported by the National Natural Science Foundation of China under Grant No.51209133, the Foundation of Shanghai Maritime University (No.20121051), Program for Innovative Team in Shanghai Maritime University, Shanghai Municipal Education Commission First-class Disciplines Project and Innovation Project of Shanghai Graduate Education (No. 20131129).

\section{REFERENCES}

1. Axworthy,D.(1997) Water distribution network modelling from steady state to waterhammer. Ph.D. thesis, Dept. of Civil Engineering,Univ of Toronto, Toronto.

2. Betamio de.Almeida.A and Koeile.E.(1992).Fluid transients in pipe networks.Computational Mechanics Publications.Southampton.C.K.

3. Cheong,L.C.(1991).Unaccounted for water and economics of leak detection.8th Int.Water Supply Congress and Exhibition.Water Suppply:The review journal of the Int. Water Supply.

4. Karney B.W.. and Mclnnis.D.(1990). Transient analysis of water distribution system. J. Of -4 WW4,82(7).62-70.

5. Ligget J.A. and Chen,L.C.(1994).Inverse transient analysis in pipe network.J Hyd.Eng.120(8),p934-955.

6. Pudar,R.S. And Liggett,J.A.(1991).Leaks in pipe networks.J of Hyd.Eng. 118(7),1992,p 1031-1046.

7. Vitkovsky,J.P. Simpson,A.R.,and Lambert,M.F.(2000). Leak detection and calibration using transients and genetic algorithms,J. Water Resour. Plann. Manage.126(4),p262-265.

8. Wylie E.B. and Streerer V.L.(1993).Fluid transients in systems.Prentice-Hall Englewood Cliffs,N.J.

\section{CONTACT WITH AUTHOR}

JunHua Li

Lzj912@163.com

YanTing Fan

School of Ocean Science and Engineering, Shanghai Maritime University Shanghai 200136

China 